\title{
Dynamic range extension of a SPAD imager using non-uniformity correction techniques
}

\author{
Eva Vilella, José García, Oscar Alonso, and Angel Diéguez, Member, IEEE
}

\begin{abstract}
The extraordinary sensitivity of single-photon avalanche diodes (SPADs) makes these devices the ideal option for vision systems aimed at low-light applications. Nevertheless, there exist large dark count rate (DCR) and photon detection probability (PDP) non-uniformities, which reduce the dynamic range of the detector. As a result, the capability to create image contrast is severely damaged or even lost. This article presents the implementation of a correction algorithm to compensate for the mentioned non-uniformities and thus extend the contrast of the generated images. To demonstrate its efficiency, the proposed technique is applied to real images obtained with a fabricated SPAD image sensor. An increase of more than 3 bits of contrast is obtained.
\end{abstract}

Index Terms-Avalanche photodiodes, CMOS integrated circuits, image processing, image sensors, optical imaging, optimization methods.

\section{INTRODUCTION}

$S_{\text {in }}$ ingle photon avalanche diodes (SPADs), especially those fabricated in CMOS technologies, have gained in recent years remarkable importance in the field of image sensors. They offer several advantages over traditional imagers, such as single-photon resolution and ultrafast response time [1]-[3]. In spite of this, SPADs suffer from noise effects and nonuniformities like all sensors.

The two most important parameters that characterize the response of a SPAD are the noise, measured mainly as dark count rate (DCR), and the sensitivity, measured as photon detection probability (PDP). The DCR is induced primarily by carriers thermally generated in the high field region of the device and also by band-to-band tunnelling to a lesser extent. Dark counts cannot be distinguished from photon triggered avalanches and may even blind the sensor [4]. The PDP is the probability that an impinging photon triggers an avalanche breakdown. Both the DCR and the PDP depend on the voltage

Manuscript received on April 23, 2015. This work was supported in part by the Spanish National Program for Particle Physics under Grant FPA201021549-C04-01.

E. Vilella was with the Department of Electronics, University of Barcelona, Martí i Franquès 1, 08028 Barcelona, Spain. She is now with the Department of Physics, University of Liverpool, Oliver Lodge, Oxford Street, Liverpool L69 7ZE, United Kingdom (e-mail: vilella@hep.ph.liv.ac.uk).

J. García was with the Department of Electronics, University of Barcelona, Martí i Franquès 1, 08028 Barcelona, Spain. He is now with the Institute of Photonic Sciences, Mediterranean Technology Park, 08860 Castelldefels, Spain.

O. Alonso and A. Diéguez are with the Department of Electronics, University of Barcelona, Martí i Franquès 1, 08028 Barcelona, Spain. over breakdown that is applied to the sensor and on the temperature. Arrays with a large number of pixels, necessary to achieve a good optical resolution, are affected by breakdown voltage variations. Because a single common high voltage is generally used to bias all the pixels of an array, i.e., the overvoltage is not the same for all the photodiodes, the DCR and the PDP are non-uniform [5]-[7]. The DCR presents a quadratic dependence with the overvoltage, whereas the PDP presents a linear behaviour prior to saturation.

Apart from that, there exist other issues which also contribute to the sensor non-uniformity. The DCR may present large variations amongst the pixels of an array due to the extreme sensitivity of SPADs to punctual defects, which are unavoidably introduced during the fabrication process. Moreover, the PDP non-uniformity can be increased as a result of the non-constant electric field across the active area of the device [8]-[9]. Finally, at high rate environments, selfheating effects may become significant and even impair the response of the sensor with irradiance.

The DCR and PDP uniformity play a key role in the dynamic range of the detector and thus on the contrast of the depicted images. So far, a few non-uniformity correction techniques for image sensors, mainly charge coupled devices (CCDs) and CMOS-active pixel sensors (CMOS-APS), have already been proposed. Most of these correction techniques are based on simple dark current subtraction to obtain a dark current corrected image frame [10]-[12]. Alternatively, correlated double sampling (CDS) can be performed to acquire at the beginning of each integration time a new and accurate dark reference [13]. Although dark current calibration is necessary, it is insufficient. Other methods that also provide gain compensation have been reported, nevertheless they rely on plain linear [14] or polynomial correction [15].

In this paper, a correction algorithm that can extend the dynamic range of an image sensor array is presented. The algorithm has been custom developed for the SPAD technology, which is well known for its exceptional sensitivity but also for its high DCR and PDP disparities [5]-[7]. However, the benefits of applying correction techniques to SPAD image sensors remain still unexplored. To the best of our knowledge, a complete correction technique is applied for the first time to compensate for both the DCR and PDP nonuniformities of a SPAD image sensor [16]. 


\section{SET-Up DESCRIPTION}

The optical set-up used to measure the DCR and PDP nonuniformities of a SPAD imager is schematically depicted in Fig. 1. The set-up is based on an illumination-optical system comprising an illumination ring with 9 white LEDs (model SMLP12WBC7W by Rohm Semiconductor) and an aspherical lens (model 352150 by Thorlabs) to focus the light on the sensor. The SPAD imager used in this experiment is an array of $10 \mathrm{x} 43$ pixels. It is monolithically integrated in the $0.35 \mu \mathrm{m}$ HV-CMOS process from ams AG. Each pixel includes a SPAD sensor and a readout circuit. A cross-section of the sensor and a diagram of the readout circuit are depicted in Fig. 2 and Fig. 3, respectively. The photodiodes are based on a pn junction, which is reverse biased above its breakdown voltage to operate in Geiger mode. The readout circuits consist of active reset $\left(\mathrm{M}_{\mathrm{N} 0} / \mathrm{RST}\right)$ and active inhibition $\left(\mathrm{M}_{\mathrm{P} 0} / \mathrm{INH}\right)$ transistors, a CMOS inverter $\left(\mathrm{M}_{\mathrm{P} 1}-\mathrm{M}_{\mathrm{N} 1}\right)$, a 1-bit memory cell $\left(\mathrm{M}_{\mathrm{N} 2}-\mathrm{M}_{\mathrm{P} 2}-\mathrm{M}_{\mathrm{N} 3} / \mathrm{CLK} 1\right)$ and a pass gate $\left(\mathrm{M}_{\mathrm{N} 4} / \mathrm{CLK} 2\right)$. Each pixel provides a one individual digital output and therefore the pixel output is expressed in counts. The reset and inhibit transistors allow to operate the SPAD array in a time-gated mode, so that in triggered applications the probability to detect dark counts can be reduced. A complete description and characterization of the SPAD array can be found in [17]. For this particular experiment, discrete measurements with gatedon and gated-off intervals of $10 \mathrm{~ns}$ and $1 \mu$ s were used. With gated-on periods of $10 \mathrm{~ns}$, the sensor is fully efficient [18]. The number of repetitions of the gated-on and gated-off cycle was $10 \cdot 10^{6}$ times. Therefore, the total measuring time was 100 $\mathrm{ms}$ for each of the points investigated. The reverse bias overvoltage of the photodiodes was set at $1 \mathrm{~V}$ throughout the whole experiment, which according to our analysis is the optimum working overvoltage of the sensor, and at room temperature.

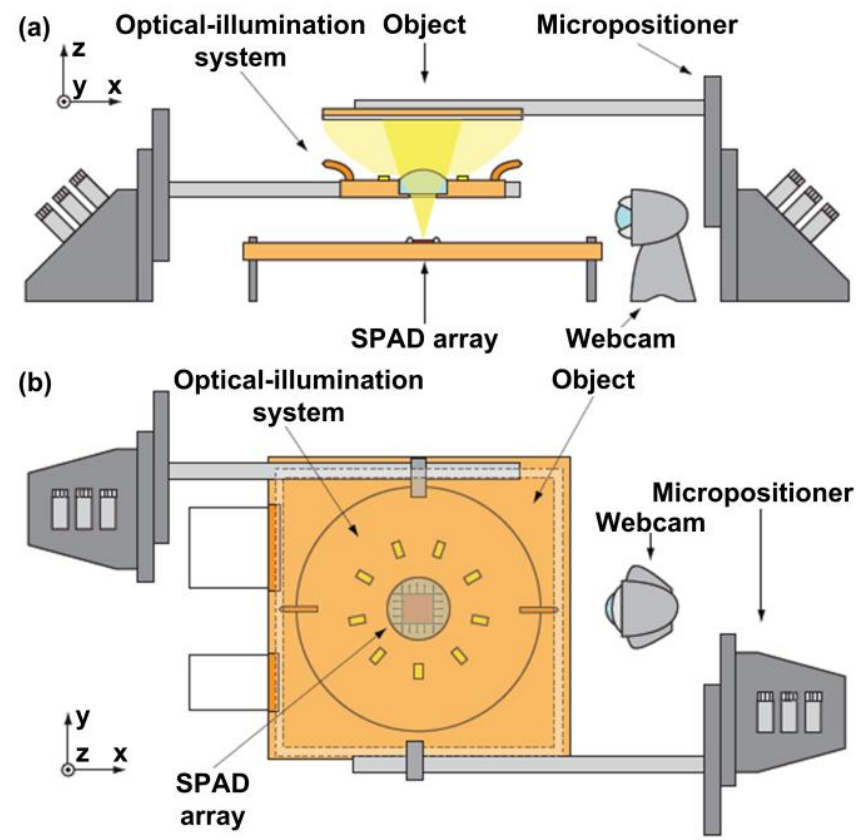

Fig. 1 Optical set-up for the measurement of the dark count and sensitivity non-uniformities. (a) Side and (b) top views.
To test the efficiency of the proposed correction method, bidimensional images of a target object placed at an appropriate distance of the sensor and optical-illumination system were taken. The images were acquired and postprocessed by means of a LabVIEW software especially developed for this purpose. Micropositioners were used to align the sensor, lens and target object. The optical set-up was placed inside a black box to avoid uncontrolled light sources.

\section{CORRECTION METHODS}

To depict a digital image, vision systems scale the range between the minimum and maximum possible values generated by the pixels of an array to a certain number of linearly spaced bins. Each bin is assigned to one representation level or colour. Typically, the lowest representation level corresponds to darkness and the maximum to saturation. However, in SPAD imagers the lowest representation level is constrained by the DCR and, as a consequence of the DCR and PDP non-uniformities, each pixel presents a different response. In addition to that, because the DCR and PDP are temperature dependent, their values may present variations at high irradiances due to self-heating effects. All these non-idealities may seriously reduce the number of representation levels that in principle is available for quantization.

This phenomenon is illustrated in Fig. 4, where the highest and lowest pixel outputs of an array are schematically depicted as a function of the irradiance. Both the pixel outputs and the

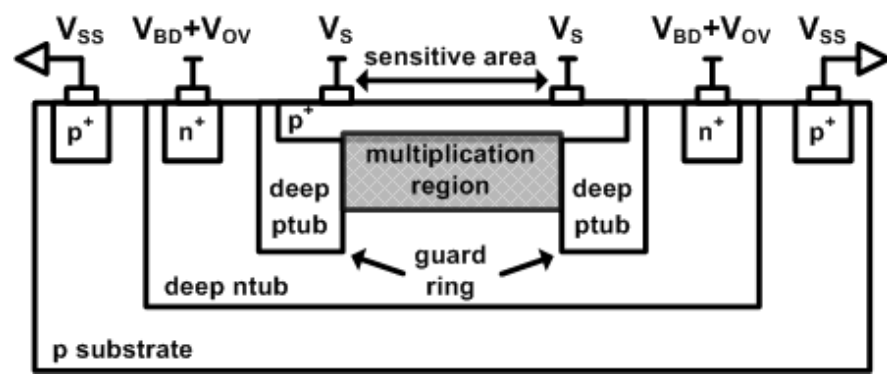

Fig. 2 Cross-section of the SPAD sensor. $\mathrm{V}_{\mathrm{BD}}$ is the breakdown voltage of the junction and $\mathrm{V}_{\mathrm{OV}}$ the applied reverse overvoltage.

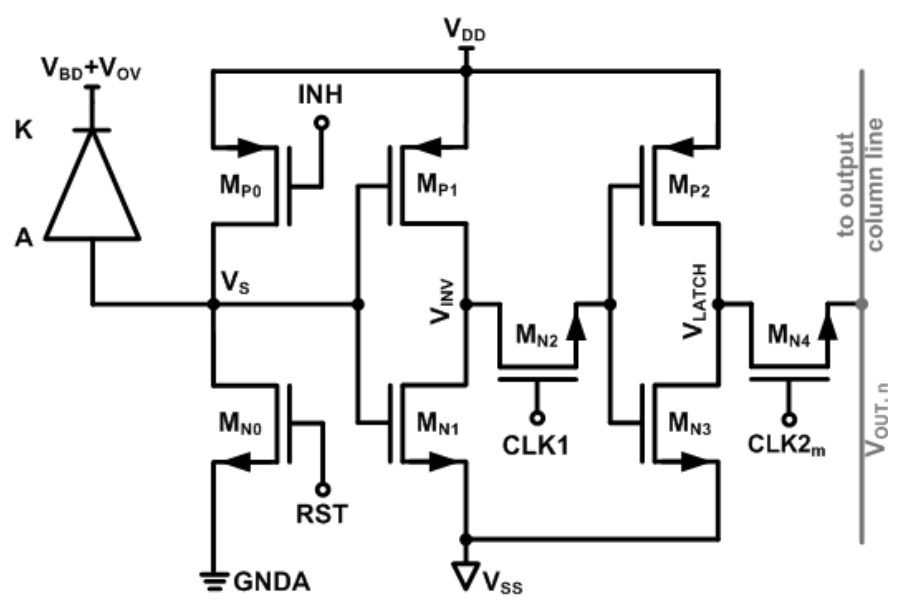

Fig. 3 Schematic diagram of the readout circuit. The signals INH, RST and CLK1 control the time-gated operation. The signal CLK2 $\mathrm{m}$ allows to sequentially read the array row by row. $\mathrm{V}_{\text {Out,n }}$ connects all the pixels of the same column. 
irradiance are split into 16 representation levels to achieve 4 bits of contrast. However, given the non-idealities of the array, the pixel outputs generated under the same irradiance are different. In fact, the differences may be so large, that the outputs generated at one particular irradiance may belong to two different representation levels. It is the case, for instance, of output levels 7 and 12 at irradiance level 9. When the highest pixel output of one level is larger than the lowest pixel output of the next level, it is impossible to establish a univocal relation between pixel outputs and irradiance levels. This results in the reduction of the representation levels that can be used to depict an image, and thus less contrast is obtained. Nevertheless, it is possible to mitigate the impact of the imager non-idealities by means of correction techniques.

The pixel counts generated by the SPAD array as a function of the power consumption of the illumination ring, which is proportional to irradiance, are shown in Fig. 5. It can be observed that the general trend of the pixel responses is to increase with irradiance, from darkness to saturation. However, the output of each pixel is different, showing a high deviation across the array that is around the $6 \%$ of the mean value. In addition, the pixel outputs differ from the expected linear behaviour, which suggests the presence of temperature effects. The breakdown voltage of the sensor increases with higher temperatures, which implies a reduction of the effective overvoltage of the photodiodes. For all these reasons, the application of a correction technique is well justified. Nevertheless, because of the high deviation, the correction method cannot be applied with equality to all the pixels and therefore it is established that a pixel-by-pixel compensation must be used.

As a first solution, a correction method that equalizes the response of all the pixels to one particular curve by using one linear equation per pixel is applied. This method assumes that the response of a certain pixel with coordinates $m$ (row) and $n$ (column) can be expressed as

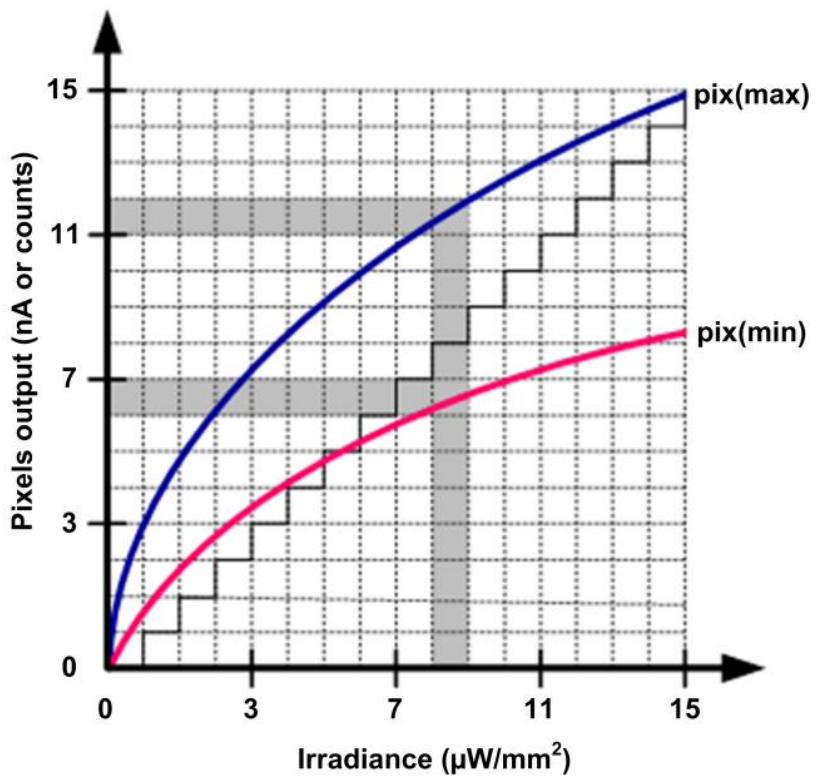

Fig. 4 Schematic plot of the the minimum (in pink) and maximum (in blue) number of counts generated by the less and most active pixels of an image sensor array.
$P C_{m n}^{k}\left(V_{O V}, T\right)=D C_{m n}\left(V_{O V}, T\right)+P D P_{m n}\left(V_{O V}, T\right) I^{k}$

where $P C_{m n}^{k}$ is the total number of counts generated by the pixel at one particular irradiance $\mathrm{k}, \mathrm{DC}_{\mathrm{mn}}$ the dark counts, $\mathrm{PDP}_{\mathrm{mn}}$ the photon detection probability and $\mathrm{I}^{\mathrm{k}}$ the incident light at that particular irradiance $\mathrm{k}$. In parentheses, $\mathrm{V}_{\mathrm{OV}}$ is the reverse overvoltage and $\mathrm{T}$ the temperature. In turn, the photon detection probability and the irradiance are given respectively by

$$
\begin{aligned}
& P D P_{m n}\left(V_{O V}, T\right)=Q E(\lambda) P_{\text {trigger }, m n}\left(V_{O V}, T\right) \\
& I^{k}=(1-R) p h_{i}
\end{aligned}
$$

where $\mathrm{QE}$ is the quantum efficiency, $\lambda$ the photon wavelength, $\mathrm{P}_{\text {trigger,mn }}$ the probability to trigger an avalanche breakdown, $\mathrm{R}$ the reflection index and $\mathrm{ph}_{\mathrm{i}}$ the number of photons emitted by the ring of LEDs that impinge the surface of the detector during the gated-on periods. To eliminate the effect of the DCR variation, the noise counts obtained in darkness are subtracted to the total pixel counts. This is done for each pixel and measured irradiance. However, frames with dark count correction only lack of significant improvement in comparison to those that are not corrected, as it can be observed in Fig. 6 (a) and Fig. 6 (b). Therefore, the idea of PDP compensation is reinforced.

PDP compensation can be achieved by applying per pixel a correcting factor to a dark count corrected frame. To calculate the correcting factor, the following equation can be used

$$
\begin{aligned}
\alpha_{m n}^{k}= & {\left[P C_{m n}^{k}\left(V_{O V}, T\right)-D C_{m n}\left(V_{O V}, T\right)\right]^{-1} } \\
& \cdot \frac{1}{i \cdot j} \sum_{m=1}^{i} \sum_{n=1}^{j}\left[P C_{m n}^{k}\left(V_{O V}, T\right)-D C_{m n}\left(V_{O V}, T\right)\right]
\end{aligned}
$$

where $\mathrm{i}$ and $\mathrm{j}$ are the maximum number of rows and columns of the array. In (4), the average pixel output across the whole array, dark count corrected and at one particular irradiance, is divided by the output of one particular pixel, also dark count

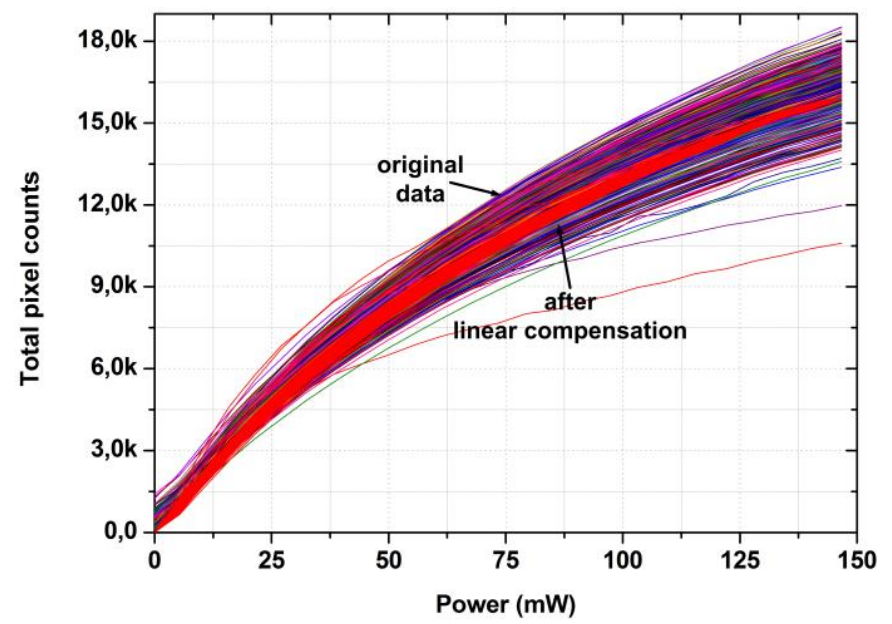

Fig. 5 Total pixel counts as a function of the power consumption of the illumination ring before (in colors) and after linear compensation (in red). 
corrected and at the same irradiance. The correcting factor will be different for each pixel. The corrected pixel counts for all the pixels can be obtained by simply

$$
P C_{m n}^{\prime}\left(V_{O V}, T\right)=\alpha_{m n}\left[P C_{m n}\left(V_{O V}, T\right)-D C_{m n}\left(V_{O V}, T\right)\right] \text {. }
$$

As a result, the response of all the pixels throughout the whole measured irradiance range is shifted to approximately the average response of the SPAD imager. In addition, because the pixel counts used to calculate the correcting factor correspond to the maximum irradiance, the DCR and PDP corrected frame presents perfect uniformity at high irradiations but this is not necessarily true for other frames obtained at other irradiations. This phenomenon can be observed in the spatial maps of Fig. 6 (c) and Fig. 6 (d). The corrected curves of all the pixels over the measured irradiance range are plotted in Fig. 5 (in red). It can be appreciated in this figure that the deviation is significantly reduced when compared to the original data (from $6 \%$ to $1 \%$ ). As a result, the number of representation levels that are available for digitization, and thus the bits of contrast, are increased. The 3.8 bits obtained with the original data are expanded to 6.9 bits after the correction. The number of bits is calculated as the base 2 logarithm of the ratio between the mean value of the counts generated by all the pixels of the array and its standard deviation. Nevertheless, more accurate results may be obtained by means of a correction algorithm that makes use of more realistic equations to homogenize the response of the pixels.

As a next step, the capabilities of a correction method that uses B-splines are investigated. A B-spline function is a piecewise polynomial approximation, where polynomials of different orders are applied to different intervals of data points and thus extremely good results can be achieved. The condition that applies is that the final value of one interval has to be the first one of the next interval. The original data plotted in Fig. 5 is split into 3 different intervals. To generate these intervals, four experimental pairs are chosen. One pair corresponds to darkness conditions and another one to the maximum measured irradiance. The other two pairs are distributed in between the irradiance range. A B-spline fit of an average pixel is shown in Fig. 7. The curve fitted points are used to generate 256 interpolated representation levels, which are equally distributed between the minimum and maximum measured irradiances. The lowest pixel output is assigned to level 0 (the darkest black), the following pixel output to level 1 , and so on until the highest value is assigned to level 255 (the brightest white). The values generated for each pixel are saved in a look-up table (LUT) that is later used to reproduce digital images. Since the response of SPADs is dependent on the overvoltage and the temperature, one different LUT for each overvoltage and temperature of interest is required. Here, we present the results obtained at $1 \mathrm{~V}$ of overvoltage and at room temperature as a demonstration of the capabilities of the method.

\section{EXPERIMENTAL RESULTS}

To show the advantages of the correction method that uses B-spline interpolated values, bidimensional images of a target object are acquired and post-processed by means of a LabVIEW software. It can be observed in Fig. 8 that the contrast and quality of the generated images are highly increased after the correction with the B-spline based algorithm.

Due to their extraordinary high sensitivity, SPAD image sensors have significant advantages over other state-of-the-art sensors, which makes them especially suitable devices for low-light applications such as fluorescence lifetime imaging microscopy (FLIM). However, the high DCR and PDP nonuniformities typical of SPAD arrays reduce severely the dynamic range of the detector. It has been demonstrated in this article that non-uniformities can be compensated in great part by means of a pixel-by-pixel correction method that uses a LUT of B-spline interpolated values.
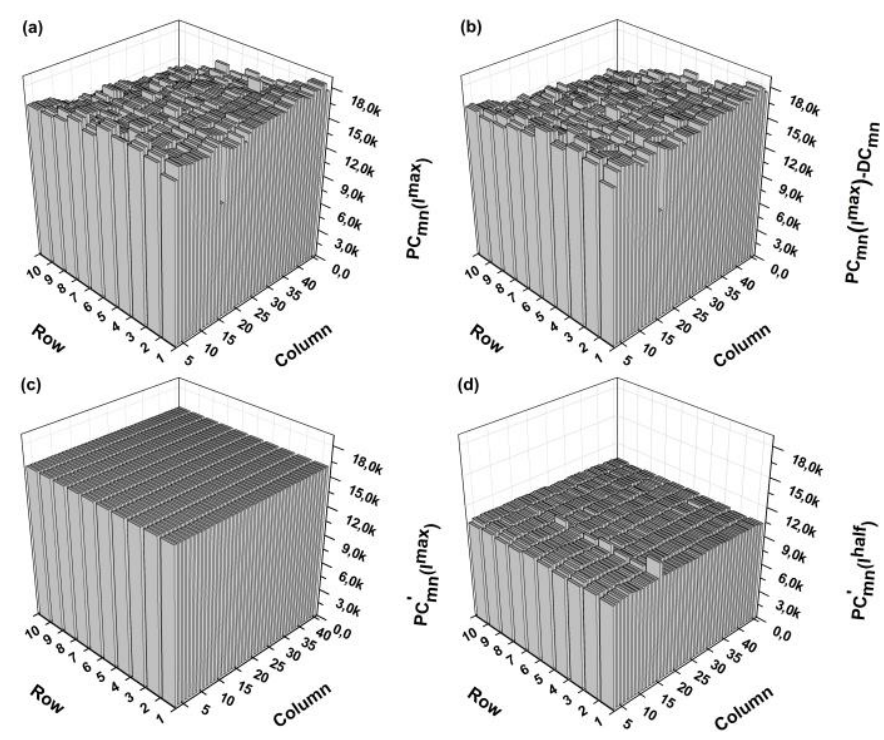

Fig. 6 (a) Total pixel counts measured at maximum irradiance, (b) total pixel counts measured at maximum irradiance minus the dark counts, (c) corrected counts at maximum irradiance and (d) corrected counts at half irradiance.



Fig. 7 B-spline fit with three different intervals (straight line) of an average pixel (dots). 


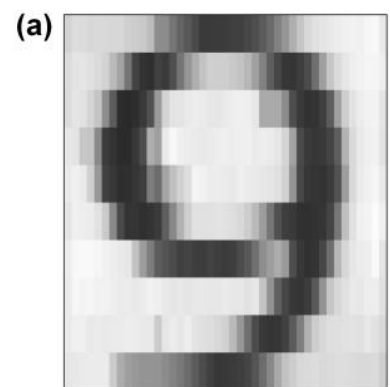

(b)

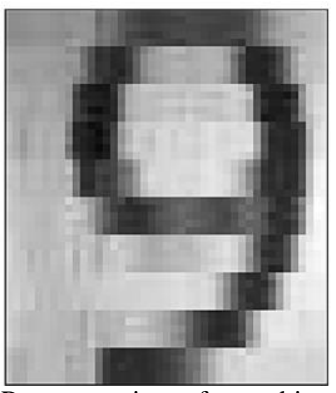

Fig. 8 Representation of an object after correction with (a) the linear algorithm and (b) the LUT based algorithm.

\section{CONCLUSION}

A novel method to correct the DCR and PDP nonuniformities in SPAD image sensors has been reported for the first time. Given the high disparities typical of SPAD arrays, the correction technique must be applied individually pixelby-pixel. Also, because of the non-linear dependency of the response of the imager with irradiance, the most accurate results can only be obtained by using B-spline interpolated values. The new correction technique increases the contrast of the depicted images.

\section{REFERENCES}

[1] A. Rochas, M. Gani, B. Furrer, P.A. Besse, R.S. Popovic, G. Ribordy, "Single photon detector fabricated in a complementary metal-oxidesemiconductor high-voltage-technology", Rev. Sci. Instrum., vol. 74, no. 7, pp. 3263-3270, Jul. 2003.

[2] F. Zappa, S. Tisa, A. Tosi, et al., "Principles and features of singlephoton avalanche diode arrays", Sens. Actuators A, vol. 140, no. 1, pp. 103-112, Oct. 2007.

[3] S. Tisa, F. Zappa, A. Tosi, et al., "Electronics for single photon avalanche diode arrays", Sens. Actuators A, vol. 140, no. 1, pp. 113-122, Oct. 2007.

[4] E. Vilella, A. Diéguez, "Avoiding sensor blindness in Geiger mode avalanche photodidoe arrays fabricated in a conventional CMOS process", J. Instrum., vol. 6, C12005, Dec. 2011.

[5] C. Veerappan, J. Richardson, R. Walker, et al., "Characterization of large-scale non-uniformities in a 20k TDC/SPAD array integrated in a 130nm CMOS process", in Proc. Solid-State Device Res. Conf., Sep. 2011, pp. 331-334.

[6] Y. Maruyama, J. Blacksberg and E. Charbon, "A time-resolved 128x128 SPAD camera for laser Raman spectroscopy”, in Proc. SPIE, Apr. 2012, pp. $83740 \mathrm{~N}$.

[7] M. Gersbach, Y. Maruyama, R. Trimananda, et al., "A time-resolved, low-noise single-photon image sensor fabricated in deep-submicron CMOS technology", IEEE J. Solid-State Circuits, vol. 47, no. 6, pp. 1394-1407, Jun. 2012.

[8] R. J. McIntyre, "On the avalanche initiation probability of avalanche diodes above the breakdown voltage", IEEE Trans. Electron Devices, vol. 20, no. 7, pp. 637-641, Jul. 1973.
[9] W. O. Oldham, R. R. Samuelson, P. Antognetti, "Triggering phenomena in avalanche diodes", IEEE Trans. Electron Devices, vol. 19, no. 6, pp. 1056-1060, Sep. 1972.

[10] J.-Y. Wu, P-K. Lu, S.-D. Liu, "Two-dimensional photo-mapping on CMOS single-photon avalanche diodes", Opt. Express, vol. 22, no. 13, pp. 16462-16471, Jun. 2014.

[11] R. L. Baer, "Efficient dark current subtraction in an image sensor", U.S. Patent 6714 241, 2004.

[12] A. Theuwissen, "Dark current correction for shutterless cameras", E.P. 2 $618560,2013$.

[13] S. K. Mendis, S. E. Kemeny, R. C. Gee, et al., "CMOS active pixel image sensors for highly integrated imaging systems”, IEEE J. SolidState Circuits, vol. 32, no. 2, pp. 187-197, Feb. 1997.

[14] S. Mohammadnejad, M. N. Sarvi and S. Roshani, "Gain and offset fixed pattern noise correction method in CCD sensors", Int. J. Nat. Eng. Sci., vol. 6, no. 3, pp. 31-15, Apr. 2012.

[15] Teledyne Rad-Icon Imaging, "AN08: Polynomial gain correction for RadEye Sensors", 2012.

[16] E. Vilella, A. Diéguez, "Dynamic range extension of SiPM detectors with the time-gated operation", Opt. Express, vol. 22, no. 10, pp. 1200712012, May 2014.

[17] E. Vilella, O. Alonso, A. Montiel, et al., "A low-noise time-gated singlephoton detector in a HV-CMOS technology for triggered imaging", Sens. Actuators A, vol. 201, no. 15, pp. 342-351, Oct. 2013.

[18] E. Vilella, A. Diéguez, "Readout schemes for low noise single-photon avalanche diodes fabricated in conventional HV-CMOS technologies, Microelectron. J., vol 44, no. 10, pp. 941-947, Oct. 2013.

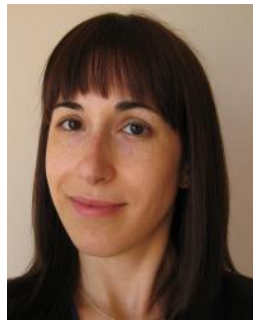

Eva Vilella received the B.Sc. and M.Sc. degrees in 2009 and 2010, respectively, both in Electronic Engineering and from the University of Barcelona. In 2013, she was awarded the Ph.D. degree in Engineering and Advanced Technologies, also from the University of Barcelona, for her work in the design, implementation and characterization of Geiger-mode avalanche photodiodes in standard CMOS technologies. The devices were aimed at the future linear colliders International Linear Collider and Compact LInear Collider. In 2014, she joined the Department of Physics at the University of Liverpool, where she is developing HV-CMOS sensors for the High-Luminosity Large Hadron Collider upgrade and future linear colliders. Her research activities are focused on the fields of ASIC design and semiconductor detectors.

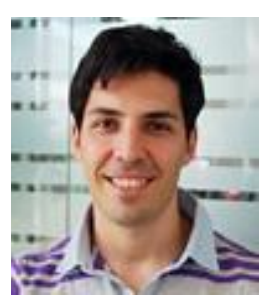

José García received the B.Sc. degree in Physics and Electronic Engineering from the University of Barcelona (UB), Spain, in 2012. He obtained the M.Sc. degree in Photonics from the Polytechnic University of Catalonia (UPC), Spain, in 2013. He is currently a $\mathrm{Ph} . \mathrm{D}$. student at the Institute of Photonic Sciences (ICFO), Spain, under the supervision of Prof. Romain Quidant. His research topics include plasmonics, microfluidics and nano-fabrication aimed at bio-sensing applications. 


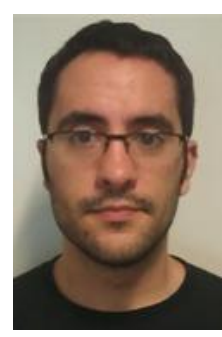

Oscar Alonso received the Ph.D. degree

in Engineering and Advanced Technologies from the University of Barcelona (UB), Spain, in 2012. Since 2014, he is a Lecturer at the Department of Electronics of UB. His research is focused on microelectronic design for microrobots and surgical-robots.

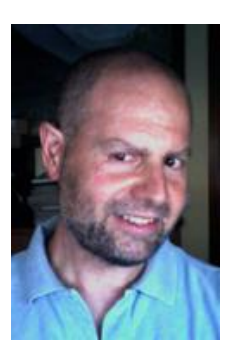

Angel Diéguez received the Ph.D. degree in Physics from the University of Barcelona (UB), Spain, 1999. Since 2001, he is an Associate Professor at the Department of Electronics of UB. His research activities have been developed mainly in the field of basic semiconductor research and microelectronic circuit design. He has participated actively in 6 European projects and has more than 40 papers. His main areas of interest are mixed-signal design of ICs and low-power VLSI design. Specific research topics are microrobotics, low-power smart sensor systems, radiation tolerant electronics and vision systems. 